\title{
Assessment of Quality of Life among Post-Menopausal Women Visited in Tertiary Care Hospital in Barmer (Rajasthan)
}

\author{
Sawai Khatri ${ }^{1}$, Balgopal Singh Bhati², Jogesh Choudhary ${ }^{3}$, Nirupama Choudhary ${ }^{3}$ \\ ${ }^{1}$ Senior Resident, Department of Obstetrics \& Gynaecology, Government Medical College, Barmer, Rajasthan, India, ${ }^{2}$ Assistant Professor, Department of Obstetrics \\ \& Gynaecology, Government Medical College, Pali, Rajasthan, India, ${ }^{3}$ Junior Resident, Department of Obstetrics \& Gynaecology, Government Medical College, \\ Barmer, Rajasthan, India.
}

\section{Abstract}

Background: Menopause is a universal event in a women's life causing a variety of physical, vasomotor, psychological and sexual symptoms. Hence; the present study was planned for assessing Quality of Life (QOL) Among Post-Menopausal Women Visited in Tertiary Care Hospital in Barmer (Rajasthan). Subjects and Methods: A total of 50 postmenopausal women were reenrolled in the present study. Postmenopausal women with more than one history of amenorrhea were included in the present study. Detailed demographic and clinical data of all the subjects was obtained. Modification of a preexisting menopause-specific QOL (MENQOL) questionnaire was used in the present study. All the items of the questionnaire were graded as present of absent. Sum total of all the symptoms comprised of QOL of postmenopausal women. All the results were recorded in Microsoft excel sheet and were analyzed by SPSS software. Results: Night sweats were found to be present in 56 percent of the subjects. Dissatisfaction with personal life and nervous feeling was found to be present in 58 percent and 8 percent of the subjects. Depression and weight gain was found to be present in 66 percent and 70 percent of the patient population. Conclusion: Menopausal significantly affects the quality of life of women. Adequate awareness programs should be started for increasing the awareness among women regarding postmenopausal symptoms.

Keywords: Post-menopausal, Quality of life, Women.

Corresponding Author: Dr. Balgopal Singh Bhati, Assistant Professor, Department of Obstetrics \& Gynaecology, Government Medical College, Pali, Rajasthan, India.

Received: April 2019

Accepted: May 2019

Introduction

Menopause is a universal event in a women's life causing a variety of physical, vasomotor, psychological and sexual symptoms. It is cessation of periods for 12 months or cessation of ovarian function resulting in permanent amenorrhoea. It usually occurs more or less in midlife, signaling the end of the reproductive phase of a women's life. ${ }^{[1-3]}$ Menopausal period is positively correlated with noncommunicable diseases such as diabetes, hypertension, osteoporosis, cervical cancer, and breast cancer. Furthermore, the menopausal women experience complex psychosocial problems - depression, mood swings, sleep disorders, loss of social, professional roles, and poor ego integrity. ${ }^{[4-6]}$ Poor compliance to recommended lifestyle modifications and limited knowledge could impede a better overall health-related quality of life (QOL). The poor QOL among high proportionate of menopausal phase of women would place a significant burden on public health care in developing countries like India. ${ }^{[5,6]}$ Hence; under the light of above obtained data, the present study was planned for assessing Quality of Life (QOL) Among Post-Menopausal Women Visited in Tertiary Care Hospital in Barmer (Rajasthan).

\section{Subjects and Methods}

The present study was conducted in the department of Obstetrics \& Gynaecology, Government Medical College, Barmer, Rajasthan, India. It included assessment of Quality of Life (QOL) Among Post-Menopausal Women Visited in Tertiary Care Hospital in Barmer (Rajasthan). Ethical approval was obtained from institutional ethical committee and written consent was obtained from all the subjects after explaining in detail the entire research protocol. A total of 50 postmenopausal women were reenrolled in the present study. Postmenopausal women with more than one history of amenorrhea were included in the present study. Detailed demographic and clinical data of all the subjects was obtained. Modification of a preexisting menopause-specific QOL (MENQOL) questionnaire, as described previously in the literature was used in the present study. ${ }^{[7]}$ All the items of the questionnaire were graded as present of absent. Sum total of all the symptoms comprised of QOL of postmenopausal women. All the results were recorded in Microsoft excel sheet and were analyzed by SPSS software.

\section{Results}

In the present study, 40 percent of the patients were more 
than 55 years of age, while 15 percent of the patients each belonged to the age group of 45 to 50 years and 50 to 55 years. In the present study, 56 percent of the subjects were educated upto level of primary education, while 12 percent of the subjects were educated upto secondary level of education. 70 percent of the patient population in the present study was residing in rural residence. Night sweats were found to be present in 56 percent of the subjects. Dissatisfaction with personal life and nervous feeling was found to be present in 58 percent and 8 percent of the subjects. Depression and weight gain was found to be present in 66 percent and 70 percent of the patient population.

\section{Number of patients}

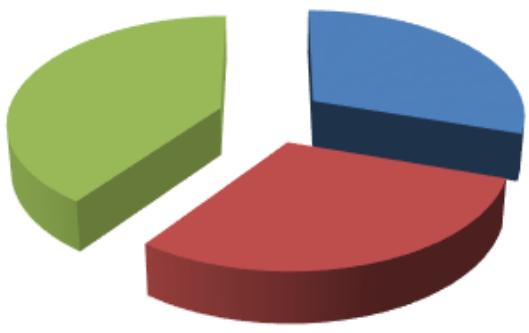

45 to 50 years

50 to 55 years

More than 55 years

Figure 1: Demographic parameter

\section{Table 1: Demographic parameter}

\begin{tabular}{|l|l|l|}
\hline Parameter & Educational \\
status & Upto primary & Number of patients \\
\cline { 2 - 3 } & Upto secondary & 28 \\
\cline { 2 - 3 } & Upto graduation & 12 \\
\cline { 2 - 3 } & Upto postgraduate & 8 \\
\hline \multirow{2}{*}{ Residence } & Rural & 35 \\
\cline { 2 - 3 } & Urban & 15 \\
\hline
\end{tabular}

Table 2: Postmenopausal symptoms among postmenopausal women

\begin{tabular}{|l|l|l|}
\hline Questions & Response & $\begin{array}{l}\text { Number of } \\
\text { patients }\end{array}$ \\
\hline \multirow{2}{*}{ Night sweats } & Yes & 28 \\
\cline { 2 - 3 } & No & 22 \\
\hline \multirow{2}{*}{ Hot flushes } & Yes & 40 \\
\cline { 2 - 3 } & No & 20 \\
\hline \multirow{2}{*}{$\begin{array}{l}\text { Dissatisfaction with } \\
\text { personal life }\end{array}$} & Yes & 29 \\
\cline { 2 - 3 } Nervous feeling & No & 21 \\
\cline { 2 - 3 } & Yes & 40 \\
\hline \multirow{2}{*}{ Poor memory } & Yes & 10 \\
\cline { 2 - 3 } & No & 29 \\
\hline \multirow{2}{*}{ Depression } & Yes & 21 \\
\cline { 2 - 3 } & No & 33 \\
\hline \multirow{2}{*}{ Vaginal dryness } & Yes & 17 \\
\cline { 2 - 3 } & No & 29 \\
\hline \multirow{2}{*}{ Dry skin } & Yes & 21 \\
\cline { 2 - 3 } & No & 33 \\
\hline \multirow{2}{*}{ Weight gain } & Yes & 35 \\
\cline { 2 - 3 } & No & 15 \\
\hline
\end{tabular}

\section{Discussion}

Menopause is defined as generally cessation of periods for 12 months or a period equivalent to three previous cycles or as time of cessation of ovarian function resulting in permanent amenorrhea. It is a stage when the menstrual cycle stops for longer than 12 months, and there is a drop in the levels of estrogen and progesterone, the two most important hormones in the female body. The onset of this physiological development not only marks the end of women's reproductive function but also introduces them to a new phase of life. ${ }^{[7-9]}$

Quality of life is a broad, multidimensional concept that lacks a precise definition in the medical literature. According to the World Health Organization, quality of life is individuals ${ }^{\text {ec }}$ perception of their position in life in the context of the culture and value systems in which they live and in relation to their goals, expectations, standards, and concerns. Quality of life tends to decline in midlife women, and there is a need to determine what role, if any, symptoms commonly associated with the transition to menopause and early postmenopausal play in this phenomenon. ${ }^{[9]}$

In the present study, 40 percent of the patients were more than 55 years of age, while 15 percent of the patients each belonged to the age group of 45 to 50 years and 50 to 55 years. In the present study, 56 percent of the subjects were educated upto level of primary education, while 12 percent of the subjects were educated upto secondary level of education. 70 percent of the patient population in the present study was residing in rural residence. Mohamed HA et al assessed the menopausal related symptoms and their impact on the women"s quality of life. A convenient sample composed of 90 women at range of age between 40- 60 years was recruited from gynecological department. An interviewing sheet was designed by the researchers and menopause specific quality of life questionnaire were used to collect the data. The most severe symptoms of vasomotor, psychosocial, physical and sexual domains were, hot flushes (29\%), experiencing poor memory (48.3\%), being dissatisfied with their personal life (44.8\%), Low backache $(41.9 \%)$, and change in sexual desire $(36.8 \%)$. The overall scores of menopausal quality of life for each domain are indicated that the highest mean score in sexual domain $(3.19 \pm 1.99)$, followed by psychosocial. It can be concluded that the most severe symptoms of vasomotor, psychosocial, physical and sexual domains were, hot flushes, poor memory, dissatisfaction with personal life, low backache, and change in sexual desire. ${ }^{[10]}$ In the present study, night sweats were found to be present in 56 percent of the subjects. Dissatisfaction with personal life and nervous feeling was found to be present in 58 percent and 8 percent of the subjects. Depression and weight gain was found to be present in 66 percent and 70 percent of the patient population. Karma D et al assessed the menopausal related symptoms and their impact on the women's quality of life. 100 postmenopausal women were assessed. They used the Menopause-Specific QOL questionnaire (MENQOL) for measuring QOL in 
postmenopausal women. The mean menopausal age was $45.80 \pm 4.49$ years. The overall mean scores obtained for each domain was $3.11+1.1$ in vasomotor domain, $2.89+$ 1.3 in psychosocial domain, $2.75+1.5$ physical domain and $2.05+1.7$ in sexual domain. Our findings showed that menopausal women had worse QOL in vasomotor domain and psychosocial domain. A large proportion of postmenopausal women suffered from menopausal symptoms. The most common menopausal complaints reported by the postmenopausal women were feeling tired, decrease stamina, changes in appearance, texture or tone of skin, aching in muscle and joints and experiencing poor memory. ${ }^{[11]}$

\section{Conclusion}

Under the light of above obtained data, the authors conclude that menopausal significantly affects the quality of life of women. Adequate awareness programs should be started for increasing the awareness among women regarding postmenopausal symptoms.

\section{References}

1. Minkin MJ, Wright CV. What Every Woman Needs to Know about Menopause: The Years Before, During and After. 1st ed. New Haven, USA: Yale University Press; 1997. p. 368.
2. Kato I, Toniolo P, Akhmedkhanov A, Koenig KL, Shore R, ZeleniuchJacquotte A. Prospective study of factors influencing the onset of natural menopause. J Clin Epidemiol. 1998;51:1271-6.

3. Kakkar V, Kaur D, Chopra K, Kaur A, Kaur IP. Assessment of the variation in menopausal symptoms with age, education and working/non-working status in North-Indian sub population using menopause rating scale (MRS). Maturitas 2007;57:306-14.

4. Sharifi N, Jalili L, Khazaeian S, Nia AN. The relationship between physical activity and general health among menopausal women in Ahvaz, Iran. Electron Physician 2017;9:3639-45.

5. Poomalar GK, Arounassalame B. The quality of life during and after menopause among rural women. J Clin Diagn Res 2013;7:135-9.

6. Anderson E, Hamburger S, Liu JH, Rebar RW. Characteristics of menopausal women seeking assistance. Am J Obstet Gynecol. 1987; 156:428-33.

7. Lewis JE, Hilditch JR, Wong CJ. Further psychometric property development of the menopause-specific quality of life questionnaire and development of a modified version, MENQOL-intervention questionnaire. Maturitas 2005;50:209-21.

8. Mansikkamäki K, Raitanen J, Nygård CH, Tomás E, Rutanen R, Luoto $\mathrm{R}$, et al. Long-term effect of physical activity on health-related quality of life among menopausal women: A 4-year follow-up study to a randomised controlled trial. BMJ Open 2015;5:e008232.

9. Lock M. Ambiguities of aging: Japanese experience and perceptions of menopause. Cult Med Psychiatry. 1986;10:23-46.

10. Mohamed HA, Lamadah SM, Zamil LG. Quality of life among menopausal women. Int $\mathrm{J}$ Reprod Contracept Obstet Gynecol 2014;3:552-61.

11. Karma D, Saini S, Singh S. Quality of Life among Post-Menopausal Women in Rural area of Punjab. J Med Sci Clin Research. 2018; 6(1): 32471-32477.

Copyright: () the author(s), 2019. It is an open-access article distributed under the terms of the Creative Commons Attribution License (CC BY 4.0), which permits authors to retain ownership of the copyright for their content, and allow anyone to download, reuse, reprint, modify, distribute and/or copy the content as long as the original authors and source are cited.

How to cite this article: Khatri S, Bhati BS, Choudhary J, Choudhary N. Assessment of Quality of Life among Post-Menopausal Women Visited in Tertiary Care Hospital in Barmer (Rajasthan). Asian J. Med. Res. 2019;8(3):OG01-OG04.

DOI: dx.doi.org/10.21276/ajmr.2019.8.3.OG1

Source of Support: Nil, Conflict of Interest: None declared. 